\title{
Sensomics - basic principles and practice
}

\author{
Tomáš Vrzal*, Jana Olšovská \\ Research Institute of Brewing and Malting, Lípová 511/15, Prague, \\ Czech Republic
}

${ }^{*}$ Corresponding author: tomas.vrzal@beerresearch.cz

\begin{abstract}
The article presents basic thoughts and principles of sensomics based on available 'omic' related literature and own practical experiences of the authors. The sensomics, a member of a wider family of 'omics' technologies applied to food (foodomics), aims to describe sensory properties of foodstuffs at a molecular level. During the last few years author's research group has been focused on expansion of this field in brewing science. Strength of the sensomics is in high capability of uncovering hidden information regarding sensory properties and effects of different raw materials as well as technological procedures on sensory properties of foodstuffs and beverages. The article aims to introduce principles and steps of sensomics together with the explanation of their importance, rules and pitfalls. Finally, the practical aspects of sensomic study is presented through sensomic comparison of the influence of decoction and infusion mashing on wort composition.
\end{abstract}

Keywords: beer, chemometrics, metabolomics, principles, sensomics, sensory active compounds

\section{Introduction}

With the growth of many different 'omic' technologies during the last decades (e.g. metabolomics, lipidomics, proteomics, genomics, transcriptomics), the food science was not left behind and subgroups of metabolomics for food and beverages were established - foodomics, flavoromics and sensomics (Skov and Engelsen, 2013; Ronningen, 2018; Haseleu, 2010). The main purpose of 'omics' is a holistic view on molecules in the sample to gain an amount of extracted information. This approach is suitable in studies aiming to the understanding of a molecular composition, a function of individual components and interconnection between them in a particular system (Capozzi, 2013). Based on the studied molecules, 'omics' are divided into respective fields - metabolomics studies metabolites, lipidomics studies lipids, proteomics studies proteins, etc. In the field of food related 'omics', the sensomics and flavoromics are focused on sensory active compounds and/or compounds that are likely to indirectly influence consumer sensory perception of a given product (e.g. by sensory interactions). On the other hand, the foodomics deals with a broader range of compounds in foods, especially compounds related to nutrition, food safety, quality and health (Capozzi, 2013, Hu, 2013).

The sensomics, as the field studying compounds related to a sensory perception of food, is not only conducted for an understanding of function of sensory active compounds in a given food product but also for the identification of a set of molecules related to a sensory perception and influence of technological or other factors on a whole sensomic profile. The reason why to use a sensomic approach in a sensory related study could be described on an example of blind men and an elephant (originating from an Indian parable adopted by many authors, e.g. The Blind Men and The Elephant - a poem by John Godfrey Saxe (Poem Hunter). This parable is about six blind men who examine an elephant by their tactile sense. Every man examines a different part of an elephant and each of them comes to a different conclusion - a man examining the elephant's ear concludes that it is a fan, a man examining the elephant's tusk concludes that it is a spear, and so on (trunk - snake, 
leg - tree, tail - rope, body - wall). However, nobody concludes the fact that it is an elephant. These misinterpretations are concluded due to limited information which each observer has. An analogical situation may occur in a single analysis study where the researcher is blind to other components of a sample. In such a situation, conclusion can be done only to a specific component, however, conclusion regarding sensory properties of a given food sample cannot be done as other components of food could influence and/ or contribute to the particular sensory attribute as well as to the overall sensory perception of food. Otherwise, using the sensomic approach, where larger sets of diverse compounds are analyzed altogether, the final view on a composition of a particular food product is more representative and usually covers the most important compounds related to sensory properties. Of course, the total number of these components is limited to a current state of knowledge and analytical chemistry. Therefore, some important components for describing given properties could be missed. Consequently, detection of these unknown components is one of the challenging aims in sensomics. Finally, the single analysis situation is comparable to the parable with an elephant, however, a consumer sensory perception of a given food could be linked to the situation when the six men would not be blind, and they see that the elephant is really an elephant. In reality, consumers by their sensory sense identify the fact, for example, that beer is really beer. Sensomics should try to work the same way.

It is necessary to mention that sensomic studies must always have a comparative character. It means that it is not possible to do a sensomic study on a set of one type of samples - for example only for one beer brand - there must be another set of samples which will serve as a control or comparative group. In the same way it is not appropriate to conduct sensomics with an excessively high number of different groups of samples - for example a comparison of ten groups of beer at the same time. In spite of the latter mentioned example, this task could be managed by comparing every single group to one reference group individually.

Due to the multivariate nature, the conduction of sensomic studies necessarily requires well controlled and defined steps enabling successful evaluation of data and extraction of relevant information without excessive bias. These steps include appropriate experimental design and sampling, sufficiently precise and reproducible analytical methods together with an adequate sample sequence, and robust data analysis and interpretation. Almost each of these steps highly determines whether the study will be successful or not. The principles, importance, rules and pitfalls of each of these steps are briefly described in the following sections.

\subsection{Study type and experimental design in sensomics}

The first step before conducting a sensomic study is to plan meaningful experimental design enabling a relevant evaluation of results and capturing desirable information. The experiment could be totally corrupted without appropriate experimental design, leading to not evaluable dataset and, of course, to wasting time and money. The first point in conducting experimental design is to take into consideration the aim of the future study (What would I like to learn from the experiment?). After that, the type of a study should be specified - there are a few possibilities regarding the type of a study - an experimental or an observational study, a data-driven or a hypothesis-driven study. The specification is necessary not only for an appropriate structure of experimental design but also for a priori knowledge of what a researcher can expect from the study as well as for correct interpretation of results. And last but not least, a sample size of experimental groups and analytes of interest (targeted or untargeted analyses - in more details in section 1.2 and 1.3) should be considered (Altmäe, 2014).

In general, an experimental study is performed in a way that random samples from population are randomly assigned into two groups (or could be in more in some cases) of different treatment (e.g. a control group and a group treated by a novel technology) while other conditions are controlled. Conversely, an observational study is based on random selection of differently treated samples from population, however, other conditions are not controllable. From this distinction it is clear that general conclusions (conclusions that can be applied to a whole population) can be made only according to the results from an experimental study, not from an observational study because there can be uncontrollable and unknown factors influencing the results. Even though experimental studies are usually preferred in sensomics, observational studies are also usable because, in some cases, it is not possible to do a required treatment of samples in the meaning of an experimental study (e.g. a geographical origin of a sample).

Based on the purpose of a given study, another type can be defined - a data-driven or a hypothesis-driven one (Horgan, 2011). The purpose of a hypothesis-driven study is to confirm or reject some of previously defined hypothesis (e.g. beer fermented by yeast strain XYZ has more pleasant taste in comparison with beer fermented by yeast strain ZYX as a consequence of a specific ratio among defined compounds in beer). Otherwise, the purpose of a data-driven study is to collect data and make a conclusion from it with no explicit a priori knowledge (e.g. What is the difference in a sensomic profile of beer fermented by two different yeast strains?). Data-driven 
studies usually lead to making some hypothesis and, furthermore, to a hypothesis-driven study. Due to the informational potential, sensomic studies are more often conducted with the data-driven approach (hypothesis-generating) at first, and then it can continue with hypothesis-driven studies.

A well-designed study should also operate with three aspects of experimental design - replication, randomization and blocking. The definition of these terms and their involvement in a study can be found in literature (Jarman, 2015, Dunn, 2012). Based on the information mentioned above, it is necessary to mention that conduction of experimental design should be done in cooperation with respective chemometrician.

\subsection{Sampling and sample preparation}

The word sampling can refer to two related but different concepts: Sampling in the meaning of analytical chemistry refers to the way in which the object is sampled before the analysis to obtain a representative sample of a given object. Unlike sampling in the meaning of chemometrics, which refers to the way in which objects are selected from the population to obtain a representative sample of objects in a study. The latter should be taken into account together with experimental design planning whereas the first is performed after the treatment conduction. The sampling (in the meaning of analytical chemistry) must ensure a homogenous and representative sample in order to avoid unwanted variation among sample replicates. The sampling strategy must also avoid any sample contamination, loss and artificial generation of analytes as well as bringing any uncontrollable variability among samples. An absolutely erroneous process is when bias is introduced in the same way as experimental design is performed (e.g. a control group of samples is sampled to glass bottles while a treatment group to plastic bottles). In a such a situation, this variability can be confounded with a treatment effect during data analysis and interpretation. Samples must be stored in order to stay chemically identical before analysis as they were at the time of sampling (snapshot of a given state of a sample). This is usually achieved by freezing samples at approximately $-20^{\circ} \mathrm{C}$ or $-80^{\circ} \mathrm{C}$ and without a possibility of sample sublimation (Antignac, 2011; Hu, 2013).

Sample preparation before analysis is another crucial step in sensomics as it can contribute to total variability among samples (Hye, 2009). The main aim of a sample preparation procedure is to transfer sample matrix and/ or analytes into an analyzable form for a given analytical platform. It can usually consist of steps as sample degassing, filtering, precipitation, dilution, analyte extraction, matrix removal, sample clean-up, volume reduction, and/ or analyte derivatization. Particular sample preparation procedure depends on a type of matrix, analytes of interest, an intended analytical method and whether the analysis will be performed in a targeted or an untargeted approach. Targeted analyses are specifically focused on predefined analytes, and therefore the sample preparation procedure is usually also targeted on capturing these compounds of interest and the remaining compounds are left behind. Sample preparation for targeted analysis should be optimized and validated in order to ensure valid and reliable results. On the other hand, untargeted analyses are not focused on predefined analytes but on an entire set of compounds in a sample. This type of analysis is carried out in order to detect and semi-quantify as many compounds in a sample as possible by a selected analytical platform. Sample preparation for untargeted analysis is performed in a same way- to capture as many compounds as possible (Mastrangelo, 2015). For this reason, sample preparation is as simple as possible to avoid discrimination of some groups. Untargeted sample preparation usually consists only of sample clean-up in case of liquid samples or extraction from solid samples. In case of the following analysis by gas chromatographic methods, unspecific derivatization process is necessary. Although such a sample preparation is not possible to validate, optimization should be done to obtain a relatively robust method with sufficient reproducibility. Untargeted analyses are taken into account in situations when there is no a priori knowledge of important compounds for a given scientific task, a study should be comprehensive enough and/or when the study is also interested in novel compounds - it allows, in contrast with the targeted approach, to make an unhypothesized association between analytes and the question of the study (Savolainen, 2016).

\subsection{Analytical methods and data acquisition}

Analytical methods in sensomics could be described as an observer of a food sample (a not blind man observing an elephant). In some cases, all analytes involved in a study could be detected by only one analytical platform, however, in a reality of sensomics, many different analytical techniques are used to cover the diversity of compounds in a given food sample (this is the case especially in targeted analysis). The selection of an appropriate analytical method is mainly directed by chemical-physical properties of targeted analytes and, of course, by accessibility of the given method. Untargeted analyses are usually carried out by a single analytical instrument, however, in order to cover compounds which are discriminated by a given instrument, a complementary analytical technique can be used. For example, compounds with a higher molecular weight and/or thermally labile compounds 
are discriminated by gas chromatography (GC) (even after appropriate derivatization), thus, a sample can be analyzed also by liquid chromatography (LC) to cover these discriminated compounds.

Selection of an appropriate detection technique is also very important. Highly selective and specific detectors could be used in targeted analysis, however, not in untargeted one. Typical detection techniques in untargeted analysis are mass spectrometry (MS) and nuclear magnetic resonance spectroscopy (NMR) since they are able to detect a wide range of compounds (Sossulini, 2017). This is the reason why GC-MS, LC-MS and NMR are the most frequently used instruments in 'omic' studies. On the other hand, there is a trend to do 'omics' by high-throughput methods which are defined as rapid, simple, sensitive, robust, low-cost and high effective (Habchi, 2016). Unfortunately, this trend is generally hard to fulfil in comprehensive sensomics studies where many diverse compounds are necessary to be detected (highly polar and nonpolar volatile compounds, low and high molecular weight).

The sample sequence during the analysis should be defined in advance. It is another important factor influencing the data quality and, unfortunately, also one of the most neglected factors in sensomics studies. General but erroneous tendency in a sample sequence is to firstly measure all samples from one group, and then samples from another group. However, this approach can lead to unwanted systematic measurement bias due to instrumental signal drift, non-enzymatic analyte conversion or interaction of the sample components with surfaces of the instrument. Therefore, a randomized sample sequence is a good alternative where possible systematic variations are randomly distributed among samples. Furthermore, these systematic variations could be corrected by some type of normalization and/or quality control samples. Normalization of a peak area to internal standard is mostly necessary in vast majority of analytical methods and is sufficient for well-documented and validated methods. On the other hand, in case of insufficiently documented methods, and especially in untargeted methods, the evenly distributed quality control (QC) samples in a sample sequence are highly recommended. Detailed aspects of QC samples in 'omics' studies were described in many review articles (Broadhurst, 2018; Dudzik, 2018).

\subsection{Data analysis and interpretation}

The true core of sensomics is chemometric data analysis - it gathers all previous steps and data, brings them together and enables interpretation. Every step described above is underlying to the extraction of relevant information from the obtained data. Therefore, every step should be planned for the purpose of this final step. Poorly performed experimental design, sampling, sample preparation, analysis and data acquisition affect resulted data analysis and subsequent interpretation, and consequently, it causes false positive and/or false negative results, misinterpretations and confusion. The widespread opinion that statistics and chemometrics can deal and cope with wrongly acquired data is mistaken. This is possible only in some specific cases but not in general meaning.

The cruciality of the previous steps is based on the multivariate nature of sensomics studies. The multivariate and comprehensive view is achieved by multivariate chemometric tools, e.g. Principal Component Analysis (PCA), Hierarchical clustering (HClust), Partial Least Squares Discriminant Analysis (PLS-DA), and many others. The main purpose of using multivariate methods in sensomics is to obtain a total image of each sample based on all variables which are available from the study (the same principle as a not blind man observing an elephant). On the other hand, there are univariate methods which evaluate only one variable at a time (the principle of a blind man observing an elephant). The difference in using univariate and multivariate chemometric methods can be described by an example in Figure 1 and 2 where are two variables, $\mathrm{X}$ and $\mathrm{Y}$, measured in two groups of experimental samples (Control and Treatment). The univariate method (in this case t-test) did not evaluate any difference between Control and Treatment group, see Figure 1. However, the comparison of these groups by multivariate methods can reveal two distinct groups, see Figure 2. Plotting of variable $\mathrm{X}$ and $\mathrm{Y}$ to a two-dimensional plot was used as the simplest multivariate (in this case bivariate) tool in chemometrics. The reason why the multivariate method identified the difference between groups in this example is that it takes into consideration all variables simultaneously, not one by one. Due to the interaction between variable $\mathrm{A}$ and $\mathrm{B}$, it was possible to find a pattern that differentiates the two groups of samples (Skov and Engelsen, 2013).

In addition to concentrations of some compounds that differ among groups, the compounds without this difference are also important in sensomics as sensory properties of a given food product are composed of every food component, not only those that differs among compared groups. And more interestingly, sensory perception of these components (without a difference) can be influenced by other components of food (with a difference) through sensory interactions (synergic, antagonistic and additive effect). Therefore, the main goal of sensomic studies is not to find food components with a difference among groups of samples. Instead, the general purpose is to find a pattern that 
Figure 1 Comparison of variables $X$ and $Y$ between control and treatment sample group

A)

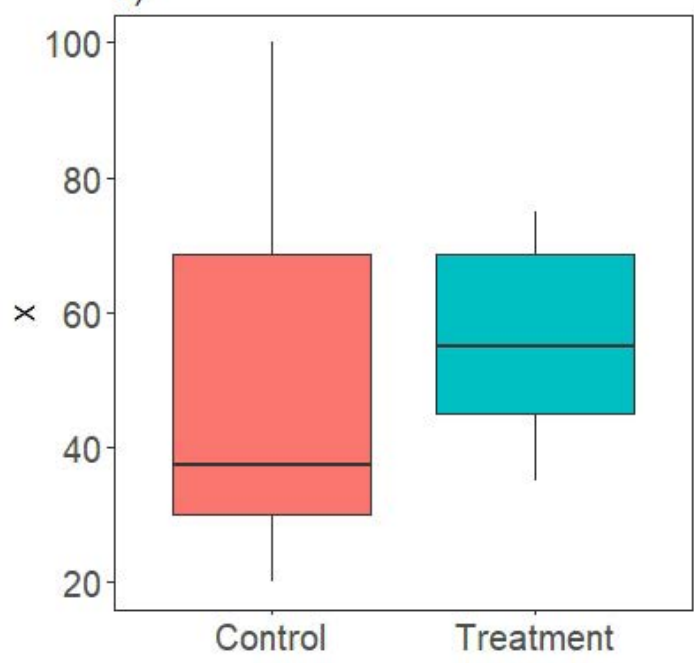

differs sample groups. On the other hand, there is a tendency in some studies to take into account only food components with concentrations above flavor threshold. This practice could be misleading from our point of view since the concept of flavor threshold is insufficient (determination of flavor threshold highly depends on concentrations of other components in sample matrix and therefore on type and brand of a given food product). Furthermore, the influence of sub-threshold compounds to an overall food flavor was described and discussed in many papers (Labbe et al., 2007).

Interpretation of results from sensomic studies should be done with caution and requires some degree of knowledge in a given field. Avoiding dogmatizing, based on previously known and published knowledge, should also be in mind because the real strength of sensomics lies in a comprehensive view to the given scientific question which was not done in the past. However, when a new finding strongly goes against the recent state of the art, it should be rigorously verified by an independent experiment. Due to the data-driven nature of majority of sensomic studies, the interpretation often leads to a hypothesis which could be verified in a subsequent experiment focused primarily on the given scientific question. Next, a possibility of confounding variables in sensomic studies should be taken into account during the interpretation. And finally, the researcher should also keep in mind that the current state of sensomics is limited by knowledge of chemistry and components of food products and the findings in new food components are the most challenging parts in sensomics to the future.
B)
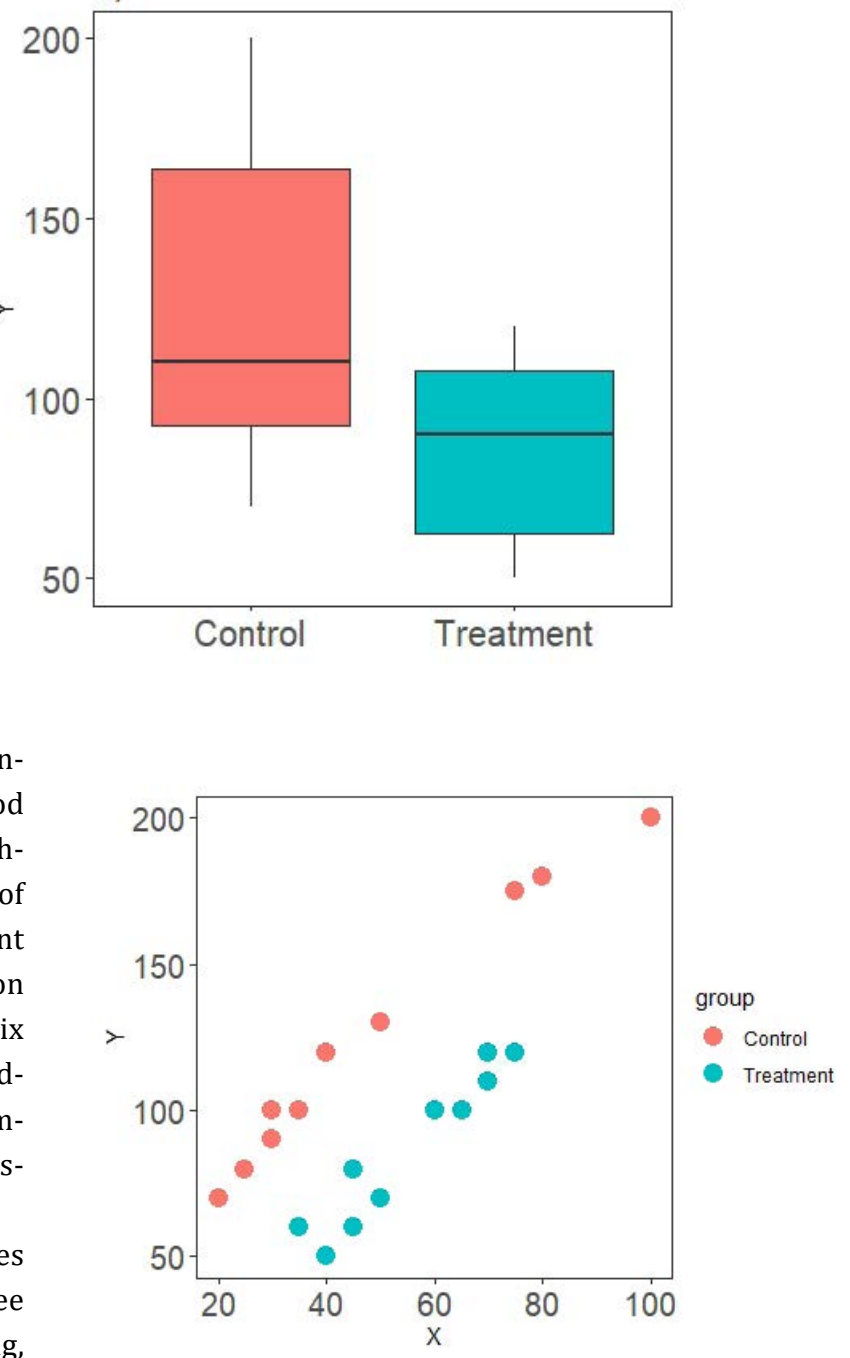

Figure 2 Multivariate (bivariate) comparison of control and treatment sample group

\section{Practical example of sensomic study}

As a practical example, the sensomic comparison of the influence of decoction and infusion mashing on wort composition was selected by the authors. This example comes from an experimental study performed at Research Institute of Brewing and Malting. The experiment consisted of wort samples with previous decoction and infusion mashing (each brewed four times). Malt (two barley varieties: Francin and Overture) from two different malt houses (marked as A and B) was used as the raw material. Factors of barley variety and malt house were blocked in the experimental design. The overview of the experimental design is shown in Table 1. The study was conducted as a targeted data-driven experiment. Wort samples were analyzed by internal methods (IM) of Re- 
search Institute of Brewing and Malting. Targeted parameters were saccharides (Jurková at al., 2014), amino acids (IM), heterocyclic compounds (IM), carbonyls (Čejka et al, 2013), total polyphenols (EBC 9.11), dimethylsulphide (EBC 9.39), bitterness of beer (EBC 9.8), fatty acids (Olšovská et al., 2019) and hop oils (IM). Concentrations of these compounds were determined by different analytical devices including spectrophotometry, gas chromatography with flame ionization and mass spectrometric detection, liquid chromatography with a refractive index and spectrophotometric detection.

Data obtained from individual analytical determinations were joined into one dataset which was subsequently evaluated by multivariate chemometric methods, particularly by HClust and PCA. HClust was performed on data standardized by z-score, Euclidean distance and a Ward method were used as a distance metric and a clustering method, respectively. The resulted dendrograms were used for construction of a heatmap (Figure 3). The first two principal components describing $67.8 \%$ from total variability in the dataset were used in PCA biplot (Figure 4) - only variables with highest loading scores are highlighted. Resulted graphical outputs from the multivariate methods (Figure 3 and 4) describe a clear separation of infusion and decoction mashed samples. The main features responsible for this separation are also graphically visible in both figures.
Table 1 Experimental design for sensomic comparison of the influence of infusion and decoction mashing on wort composition

\begin{tabular}{|c|c|c|c|}
\hline Sample No. & Malt house & Barley variety & Mashing \\
\hline 1 & A & Francin & Decoction \\
\hline 2 & B & Francin & Decoction \\
\hline 3 & A & Overture & Decoction \\
\hline 4 & B & Overture & Decoction \\
\hline 5 & A & Francin & Infusion \\
\hline 6 & B & Francin & Infusion \\
\hline 7 & A & Overture & Infusion \\
\hline 8 & B & Overture & Infusion \\
\hline
\end{tabular}

Figure 3 Heatmap representation of experimental samples

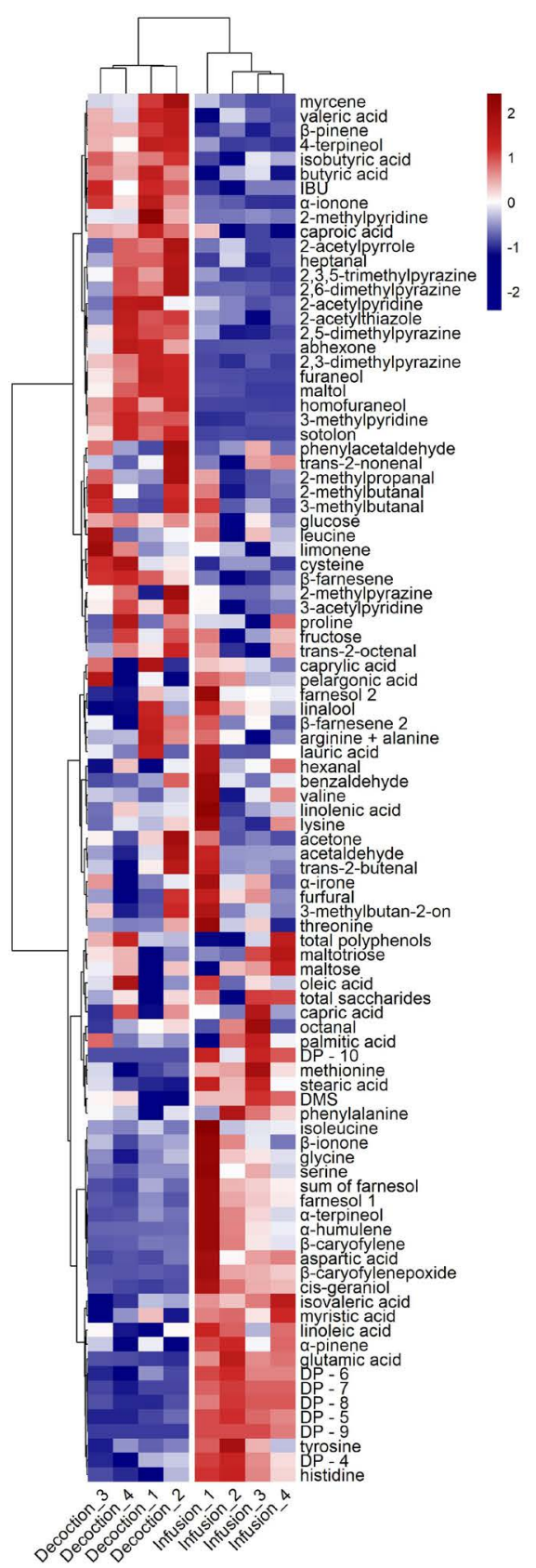


These features include heterocyclic compounds (e.g. furaneol, maltol, homofuraneol, pyridines, pyrazines and thiazoles) which are upregulated after decoction mashing. On the other hand, oligosaccharides (with degree of polymerization 4-10) were upregulated after infusion mashing. These findings are in good agreement with the basic theory of decoction mashing where more intensive enzymatic and Maillard reactions occur, therefore, it resulted in lower concentrations of oligosaccharides and higher concentrations of heterocyclic compounds.

Hop oils also significantly contribute to the clustering of the experimental samples, however, there is not one clear direction (upregulated after decoction or infusion mashing) as it was in the previously mentioned groups of compounds. In this case hop oils formed two groups - the first one is upregulated after decoction and the second one after infusion mashing. The possible mechanism of this phenomenon is different intramolecular interaction between hop oil constituents and wort constituents based on different wort composition. This result suggests a mashing process as a potential factor for controlling the final hop aroma of beer. However, this result should be perceived with caution and it should be confirmed by an independent experiment.

The basic differences of wort prepared by infusion and decoction mashing were shown in this small practical example of a sensomic study. These differences can influence composition of the final beer and therefore its sensory properties. It should be noted that also other components (without any observable difference) are important as they potentially could sensorially affect perception of other components. It must be highlighted that sensory analysis was not performed in this practical example since wort is not the final product designated for consumption, but sensomic studies should generally be completed with sensory analysis.

\section{Conclusion}

The basic principles and practical requisites of sensomics are summarized in this article. The sensomics is highly capable of uncovering hidden information regarding sensory properties and effects of different raw materials as well as technological procedures on sensory properties of foodstuffs and beverages. However, this is true only in situation when all steps are conducted correctly. The practical example of the influence of decoction and infusion mashing on wort composition reveals some interesting aspects of differences between differently mashed wort, as well as, some aspects of sensomic studies in general. Finally, the importance of careful interpretation and sub- sequent confirmation of the results was highlighted. The sensomics is quite a young field and principal studies will be done in future. The article contributes to awareness of the sensomics in scientific and brewing community.

\section{Acknowledgement}

This study was supported by Ministry of Education, Youth and Sports of the Czech Republic No. LO 1312

\section{References}

Analytica EBC, EBC Analysis Commitee-Nürnberg: Carl Getranke-Fachverlag. 2012.

Altmäe, S., Esteban, F.J., Stavreus-Evers, A., Simón, C., Giudice, L., Lessey, B.A., Horcajadas, J.A., Macklon, N.S., D’Hooghe, T., Campoy, C., Fauser, B.C., Salamonsen, L.A., Salumets, A. (2014). Guidelines for the design, analysis an interpretation of 'omics'data: focus on human endometrium. Human Reproduction Update, 20 (1), 12-28. https://doi. org/10.1093/humupd/dmt048

Antignac, J. P., Courant, F., Pinel, G., Bichon, E., Monteau, F., Elliott, C., Le Bizec, B., (2011). Mass spectrometry-based metabolomics applied to the chemical safety of food. Trends in Analytical Chemistry, 30 (2), 293-301. https://doi.org/10.1016/j.trac.2010.11.003

Broadhurst, D., Goodacre, R., Reinke, S. N., Kulingowski, J., Wilson I. D., Lewis, M. R., Dunn, W. B., (2018). Guidelines and considerations for the use of system suitability and quality control samples in mass spectrometry assays applied in untargeted metabolomic studies. Metabolomics 14 (72). https://doi.org/10.1007/s11306-018-1367-3

Cappozzi, F., Bordoni, A. (2013). Foodomics: a new comprehensive approach to food and nutrition. Genes \& Nutrition, 8(1), 1-4. https:// doi.org/10.1007/s12263-012-0310-x

Čejka, P., Čulík, J., Horák, T., Jurková, M., Olšovská, J. (2013). Use of Chemical Indicators of Beer Aging for Ex-post Checking of Storage Conditions and Prediction of the Sensory Stability of Beer. J. Agric. Food Chem. 61, 12670-12675. https://doi.org/10.1021/jf403361h

Dudzik, D., Barbas-Bernandos, C., García, A., Barbas, C. (2018). Quality assurance procedures for mass spectrometry untargeted metabolomics. a review. Journal of Pharmaceutical and Biomedical Analysis, 147, 149-173. https://doi.org/10.1016/j.jpba.2017.07.044

Dunn, W. B., Wilson, I. D., Nicholls, A. W., Broadhurst, D. (2012). The importance of experimental design and QC samples in large-scale and MS-driven untargeted metabolomic studies of humans. Bioanalysis, 4 (18), 2249-2264. https://doi.org/10.4155/bio.12.204

Habchi, B., Alves, S., Paris, A., Rutledge, D. N. (2016). How to really perform high throughput metabolomic analyses efficiently? Trends in Analytical Chemistry, 85, 128-139. https://doi.org/10.1016/j.trac.2016.09.005

Haseleu, G., Lagemann, A., Stephan, A., Intelmann, D., Dunkel, A., Hofmann, T. (2010). Quantitative Sensomics Profiling of Hop-Derived Bitter Compounds Throughout a Full-Scale Beer Manufacturing Process. Journal of Agricultural and Food Chemistry, 58 (13), 7930-7939. https://doi.org/10.1021/jf101326v

Horgan, R.P., Kenny, L.C. (2011). 'Omic'technonogies: genomics, transcriptomics, proteomics and metabolomics. The Obstetrician \& Gynaecologist, 13, 189-195. https://doi.org/10.1576/toag.13.3.189.27672

Hu Chunxiu, Xu Guowang (2013). Mass-spectrometry-based metabolomics analysis for foodomics - Review. Trends in Analytical Chemistry, 52, 36-46. https://doi.org/10.1016/j.trac.2013.09.005 
Hye, K.K., Verpoorte, R., (2009). Sample Preparation for Plant Metabolomics. Phytochemical Analysis, 21 (1), 4-13. https://doi.org/10.1002/ pca.1188

Jarman, K. H., 2015: Beyond basic statistics: tip, tricks and techniques every data analyst should know. John Wiley \& Sons, Inc. ISBN 9781-118-85611-6

Jurková, M., Čejka, P., Štěrba, K., Olšovská, J., (2014). Determination of total carbohydrate content in beer using its precolumn enzymatic cleavage and HPLC-RI. Food Anal. Met., 7(8), 1677-1686. https://doi. org/10.1007/s12161-014-9805-y

Labbe, D., Rytz, A., Morgenegg, C., Ali, S., Martin, N., (2007). Subthreshold Olfactory Stimulation Can Enhance Sweetness. Chem. Senses, 32, 205 - 214. https://doi.org/10.1093/chemse/bj1040

Mastrangelo, A., Ferrarini, A., Rey-Stolle, F., Garcia, A., (2015). From sample treatment to biomarker discovery: A tutorial for untargeted metabolomics based on GC-(EI)-Q-MS. Analytica Chimica Acta, 900, 21 - 35. https://doi.org/10.1016/j.aca.2015.10.001

Olšovská, J., Vrzal, T., Štěrba, K., Kubizniaková, P., Čejka, P., (2019). The chemical profiling of fatty acids during the brewing process. J Sci Food Agric 99(4), 1772-1779. https://doi.org/10.1002/jsfa.9369
Poem Hunter [online]. https://www.poemhunter.com/poem/the-blindman-and-the-elephant/ [2019-04-17]

Ronningen, I., Miller, M., Xia, Y., Peterson, D. G. (2018). Identification and Validation of Sensory-Active Compounds from Data-Driven Research: A Flavoromics Approach. Journal of Agricultural and Food Chemistry, 66 (10), 2473-2479. https://doi.org/10.1021/acs.jafc.7b00093

Savolainen, O. I., Sandberg, A. S., Ross, A. B. (2016). A Simultaneous Metabolic profiling and Quantitative Multimetabolite Metabolomic Method for Human Plasma Using Gas-Chromatography Tandem Mass Spectrometry. Journal of Proteome Research, 15 (1), 259-265. https://doi.org/10.1021/acs.jproteome.5b00790

Sossulini, A., 2017: Metabolomics: From Fundamentals to Clinical Applications. Springer International Publishing, ISBN 978-3-319-47656-8

Skov, T., Engelsen S. B. (2013). Chapter 19 Chemometrics, Mass Spectrometry, and Foodomics in Cifuentes, A. Foodomics: Advanced Mass Spectrometry in Modern Food Science and nutrition. First Edition, John Wiley \& Son, Inc. https://doi.org/10.1002/9781118537282.ch19 